Check for updates

Cite this: Phys. Chem. Chem. Phys., 2022, 24, 6169

Received 4th January 2022,

Accepted 15th February 2022

DOI: $10.1039 / \mathrm{d} 2 \mathrm{cp} 00042 \mathrm{c}$

rsc.li/pccp

\section{Dynamical component exchange in a model phase separating system: an NMR-based approach $\uparrow$}

\author{
Christian F. Pantoja, (D) ${ }^{a}$ Markus Zweckstetter (D) *ab and \\ Nasrollah Rezaei-Ghaleh (D) *bcd
}

\begin{abstract}
Biomolecular phase separation plays a key role in the spatial organization of cellular activities. Dynamic formation and rapid component exchange between phase separated cellular bodies and their environment are crucial for their function. Here, we employ a well-established phase separating model system, namely, a triethylamine (TEA)-water mixture, and develop an NMR approach to detect the exchange of scaffolding TEA molecules between separate phases and determine the underlying exchange rate. We further demonstrate how the advantageous NMR properties of fluorine nuclei provide access to otherwise inaccessible exchange processes of a client molecule. The developed NMR-based approach allows quantitative monitoring of the effect of regulatory factors on component exchange and facilitates "exchange"-based screening and optimization of small molecules against druggable biomolecular targets located inside condensed phases.
\end{abstract}

\section{Introduction}

Liquid-liquid phase separation has emerged as a key physicochemical principle underlying the spatial organization of biomolecules within the cell, and thereby reinvigorated the interest in studying this classical physical chemistry phenomenon. ${ }^{1-4}$ Several biophysical techniques have been developed to monitor biomolecular phase separation under in vitro and in vivo conditions and determine the structural dynamics of its constituents, including proteins, nucleic acids, ions and water molecules. ${ }^{5-12}$ A remarkable feature of phase separated cellular bodies is their rapid formation and dissolution in response to regulatory signals and dynamic component exchange with the cellular environment. ${ }^{13-15}$ The component exchange occurs not only for the socalled scaffold molecules whose phase behavior governs the formation of phase separated droplets, but also for the client molecules which enter the formed dropelts and undergo dynamic exchange with the environment. ${ }^{16-18}$ These features are particularly

\footnotetext{
${ }^{a}$ Translational Structural Biology Group, German Center for Neurodegenerative Diseases (DZNE), Von-Siebold-Strasse 3a, D-37075 Göttingen, Germany. E-mail: Markus.Zweckstetter@dzne.de

${ }^{b}$ Department of NMR-based Structural Biology, Max Planck Institute for Multidisciplinary Sciences, Am Faßberg 11, D-37077 Göttingen, Germany ${ }^{c}$ Institut für Physikalische Biologie, Heinrich-Heine-Universität Düsseldorf, Universitätsstr. 1, D-40225 Düsseldorf, Germany.

E-mail: Nasrollah.Rezaie.Ghaleh@hhu.de

${ }^{d}$ Institute of Biological Information Processing (IBI-7: Structural Biochemistry), Forschungszentrum Jülich, Wilhelm-Johnen-Straße, 52428 Jülich, Germany $\dagger$ Electronic supplementary information (ESI) available: Supplementary methods (chemicals, DIC and fluorescence imaging, NMR experiments and data analysis) and Fig. S1. See DOI: 10.1039/d2cp00042c
}

advantageous in rapidly changing environments and are crucial for the functioning of several cellular bodies. ${ }^{19-21}$

Despite its importance in physiological and potentially pathological processes, little is known about how the component exchange of membrane-less phase separated bodies is regulated by biochemical factors, e.g. mutation or chemical modifications in phase-separating biomolecules, the presence of small molecules, etc. Progress in this direction requires the development of experimental methods for the quantitative determination of exchange rates. Here, we use a model system for phase separation, namely a triethylamine (TEA)-water mixture, and develop a multi-spin NMR approach to study the exchange process between separated phases at a quantitative level. TEA is a well-established phase separation model system, which undergoes a reversible temperature-dependent phase separation, exhibiting lower critical solution temperature (LCST) behaviour. ${ }^{22-26}$ As a result of phase separation, two phases are formed: a TEA-enriched condensed phase and water-enriched phase. In addition to TEA as the scaffold molecule, we investigate the exchange of a fluorine-containing client molecule between separated phases and show how the use of ${ }^{19} \mathrm{~F}$ NMR allows detection of the exchange process and quantification of its rate.

\section{Results and discussion}

Probing TEA phase separation by microscopy and NMR

First, we examined the temperature-dependent phase separation of a mixture of TEA and dextran using microscopy. The use of dextran as a crowding agent was to slow down the kinetics of the TEA phase separation process. In addition, fluorescently 
labeled dextran was used in order to monitor the phase separation process. As reported in the literature, the waterTEA and $\mathrm{D}_{2} \mathrm{O}-\mathrm{TEA}$ mixtures have LCSTs of $c a .18$ and $15{ }^{\circ} \mathrm{C}$, respectively. ${ }^{26,27}$ As shown in Fig. 1A, at $278 \mathrm{~K}$, the TEA sample exhibited a single dispersed phase, with a nearly uniform background fluorescence emission originating from the labeled dextran. We then increased the temperature to $298 \mathrm{~K}$, resulting in the phase separation of the sample and the formation of a large number of variously sized spherical droplets with an

A
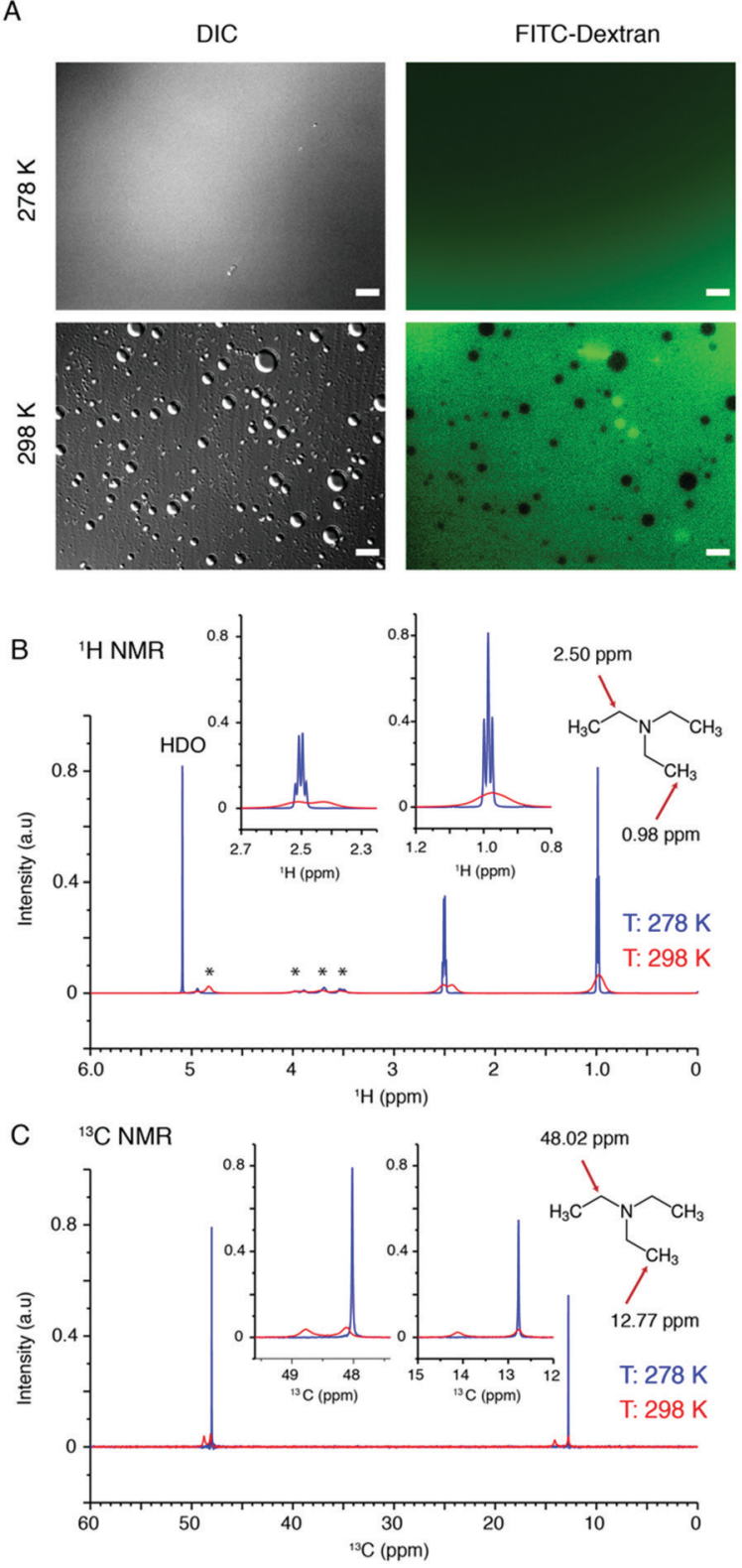

Fig. 1 Liquid-liquid phase separation of triethylamine (TEA)/water/ dextran mixture, probed by differential interference contrast (DIC) and fluorescence microscopy (A), and ${ }^{1} \mathrm{H}(\mathrm{B})$ and ${ }^{13} \mathrm{C}$ (C) NMR spectroscopy. ${ }^{1} \mathrm{H}$ signals from dextran are marked by stars in (B). The phase separationinduced broadening in TEA's methylene and methyl ${ }^{1} \mathrm{H}$ (B) and ${ }^{13} \mathrm{C}$ (C) signals, as well as the emergence of new ${ }^{13} \mathrm{C}$ signals can be seen. Scale bars, $10 \mu \mathrm{m}$. approximate average radius of $4.5 \pm 1.5 \mu \mathrm{m}$ (Fig. 1A). Notably, the dextran was almost completely excluded from the formed droplets and remained in the dispersed phase.

Next, we studied the phase separation of TEA using ${ }^{1} \mathrm{H}$ NMR. At $278 \mathrm{~K}$, where the TEA sample displays a single dispersed phase (Fig. 1A), the $1 \mathrm{D}^{1} \mathrm{H}$ NMR spectrum showed signals at $c a$. 2.5 and 0.98 ppm, which belong to its methylene and methyl protons, respectively (Fig. 1B). The narrow linewidth of the TEA ${ }^{1} \mathrm{H}$ signals reflect the large level of mobility of this small-sized molecule within the spatially homogeneous dispersed phase. After increasing the temperature to $298 \mathrm{~K}$, and the resultant phase separation, the TEA ${ }^{1} \mathrm{H}$ signals were strongly broadened, and consequently, the characteristic quartet and triplet splitting patterns of methylene and methyl signals were no longer observable. The phase separation-induced signal broadening was not limited to TEA signals, but also observed for the dextran signal. As dextran did not enter the formed droplets, the broadening of its signals seems to reflect the magnetic susceptibility inhomogeneity of the phase separated sample. Accordingly, the TEA signal broadening is potentially caused by three major factors: the altered mobility of TEA within the condensed interior of droplets, the chemical exchange between different phases, and sample inhomogeneity. In addition to signal broadening, the methylene, but not the methyl, signal showed the emergence of a new peak $\sim 0.1 \mathrm{ppm}$ upfield to the original signal.

Next, we investigated the phase separation of TEA using 1D ${ }^{13} \mathrm{C}$ NMR at natural abundance. As expected, the spectrum of the single-phase dispersed TEA sample at $278 \mathrm{~K}$ showed two sharp ${ }^{13} \mathrm{C}$ signals at $\mathrm{ca} .48 .02$ and $12.77 \mathrm{ppm}$, belonging to methylene and methyl groups, respectively (Fig. 1C). Similar to the ${ }^{1} \mathrm{H}$ signals, the temperature-induced phase separation led to a significant broadening of TEA's ${ }^{13} \mathrm{C}$ signals and a decrease in their peak height, likely due to the induced alteration in mobility, exchange and sample homogeneity. Unlike the ${ }^{1} \mathrm{H}$ spectrum, however, the ${ }^{13} \mathrm{C}$ spectrum of the phaseseparated TEA sample showed two well-resolved new signals downfield of the original methylene and methyl signals. The emergence of these new ${ }^{13} \mathrm{C}$ signals (and the new ${ }^{1} \mathrm{H}$ signal of the methylene group, see above) pointed to the presence of an exchange process between the dispersed and condensed phases of TEA, at a slow rate on the NMR chemical shift timescale, i.e. $<1300 \mathrm{~s}^{-1}$ (see Fig. 2A).

\section{NMR-based identification of the kinetic stages of TEA phase separation}

As a prerequisite for the quantitative investigation of dynamical exchange processes underlying the phase separation of TEA, we established the kinetics of this process through real-time 1D ${ }^{13} \mathrm{C}$ NMR experiments, where we monitored the methyl signals belonging to the dilute and condensed phase. Immediately after the temperature jump from 278 to $298 \mathrm{~K}$, the signal belonging to the dilute phase was significantly broadened and partially lost its peak height. However, further progression of the phase separation process led to the partial recovery of the linewidth and peak height of this signal (Fig. 2A). The broad 
A

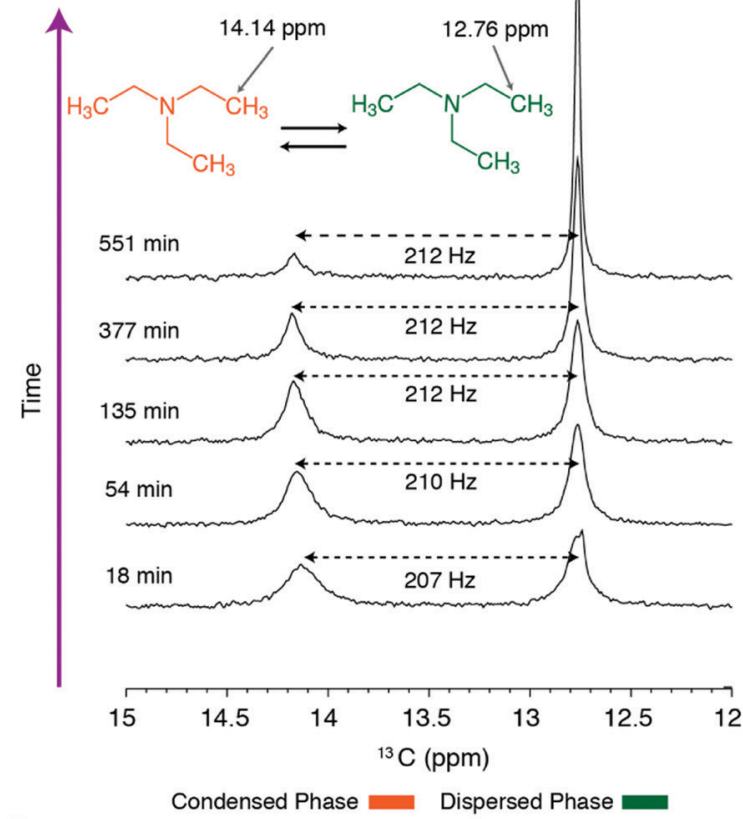

B

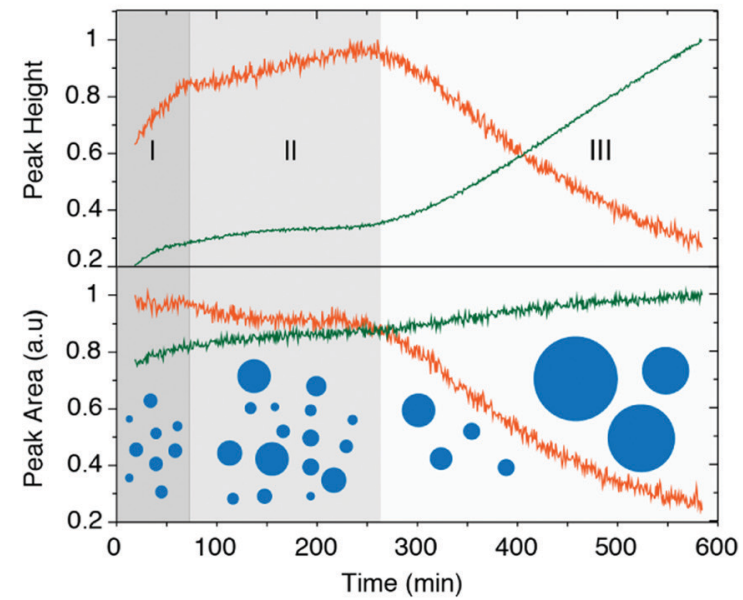

Condensed Phase Dispersed Phase

Fig. 2 Kinetics of liquid-liquid phase separation in triethylamine (TEA)/ water $\left(D_{2} \mathrm{O}\right) /$ dextran mixture, monitored through ${ }^{13} \mathrm{C}$ methyl signals of TEA molecules. (A) Changes in the $1 D{ }^{13} \mathrm{C}$ NMR spectrum of the methyl group of TEA after inducing phase separation through a temperature jump from 278 to $298 \mathrm{~K}$. The signals at 12.76 and $14.14 \mathrm{ppm}$ originate from the dispersed and condensed phase, respectively. (B) Relative peak areas of ${ }^{13} \mathrm{C}$ methyl signals of TEA from the two phases (bottom panel) and the peak heights (top panel) as seen in (A). Three kinetic stages of phase separation (I-III, shaded areas, respectively showing formation, growth and precipitation of large droplets) can be distinguished.

signal belonging to the condensed phase followed a rather distinct trend: the gradual narrowing of the signal during the first 1-3 hours of the process was followed by a sharp decrease in peak height (Fig. 2B). The time-dependence of peak heights and areas (Fig. 2B) indicated the presence of three kinetic stages: an initial stage (around 60 minutes), when the condensed phase signal grew in height, a middle stage (around 60-260 minutes) during which the peak intensities remained relatively stable, and the final stage (ca. 260-600 minutes) when a sharp intensity loss was observed for the condensed phase signal. The final intensity loss of the condensed phase signal was due to the formation of large TEA droplets and their precipitation and exit from the detection zone of NMR coils. Notably, this precipitation is reverse, i.e. the TEA-enriched phase is not at the bottom but at the top of the sample, due to the lower density of TEA-enriched droplets when compared to the TEA-depleted phase. Accordingly, during kinetic stages I and III, the dominant processes are respectively droplet formation and precipitation, while the kinetic stage II represents a quasi-steady-state during which these two processes partially cancel the effect of each other on NMR signal intensities.

\section{NMR-based investigation of the initial TEA exchange between phases}

The study of phase separation-related exchange processes during later kinetic stages (i.e. stage III in Fig. 2B) is complicated by the (reverse) precipitation-induced NMR intensity changes. To minimize the interfering effects of (reverse) precipitation, we took advantage of the reversibility of phase separation and developed an experimental scheme, where cycles of heatingcooling were employed and NMR data were collected only during the initial kinetic stage of phase separation (typically the first 15-20 minutes, depending on the experiment). Using this scheme, we were able to measure a clean $2 \mathrm{D}$ projection plane of the ${ }^{13} \mathrm{C},{ }^{1} \mathrm{H},{ }^{1} \mathrm{H}$ HSQC-NOESY spectrum of TEA during phase separation. As shown in Fig. $3 \mathrm{~A},{ }^{13} \mathrm{C},{ }^{1} \mathrm{H}$ correlation peaks were observed for TEA methylene and methyl groups from both the dispersed and condensed phases and several groups of dextran. Interestingly, the TEA ${ }^{1} \mathrm{H}$ signals belonging to the two phases showed nice correlation peaks, indicating either spatial proximity (and its consequent dipolar coupling-mediated correlation) or chemical exchange between them. The sign of cross-peaks in these and complementary $2 \mathrm{D}{ }^{1} \mathrm{H},{ }^{1} \mathrm{H}$ ROESY spectra (Fig. 3B) excluded the dipolar coupling origin and confirmed the presence of chemical exchange. Notably, no correlation peaks were observed for dextran signals, consistent with the exclusion of dextran from the TEA droplets (Fig. 1A).

Then, we employed the heating-cooling cycles and measured the $2 \mathrm{D}{ }^{1} \mathrm{H},{ }^{1} \mathrm{H}$ EXSY spectra of the phase separated sample at five different mixing times ranging from 15 to $120 \mathrm{~ms}$ (Fig. 3C). The EXSY spectra at each mixing time were collected during the first 20 minutes after the initiation of phase separation. The intensity of the exchange-induced correlation peaks displayed the characteristic dependence on mixing time. Analysis of the intensity build-up curves for the methylene and methyl groups provided an effective exchange rate, $k_{\mathrm{ex}}$, of $31 \pm 5 \mathrm{~s}^{-1}$, for the exchange of TEA molecules between two phases (Fig. 3D), i.e. an exchange process that is slow on the NMR chemical shift timescale.

\section{Fluorine NMR-based investigation of the exchange of a client molecule between TEA phases}

To further investigate the component exchange between the phase separated TEA droplets and their environment, we studied the exchange of hexafluoroisopropanol (HFiP) as a 
A
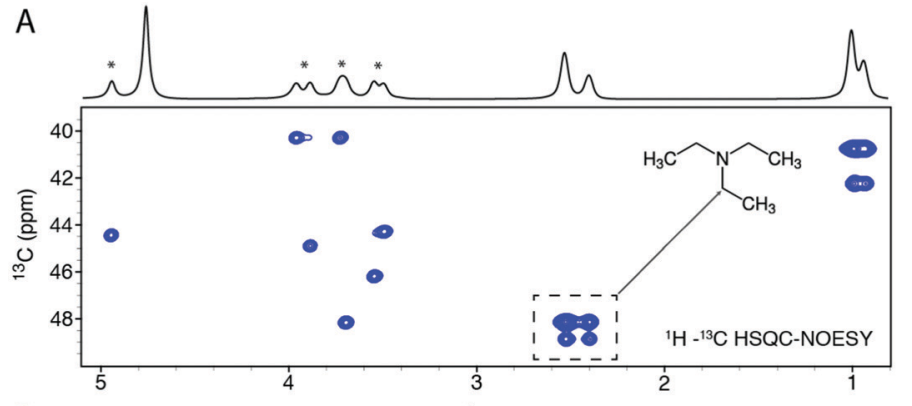

C
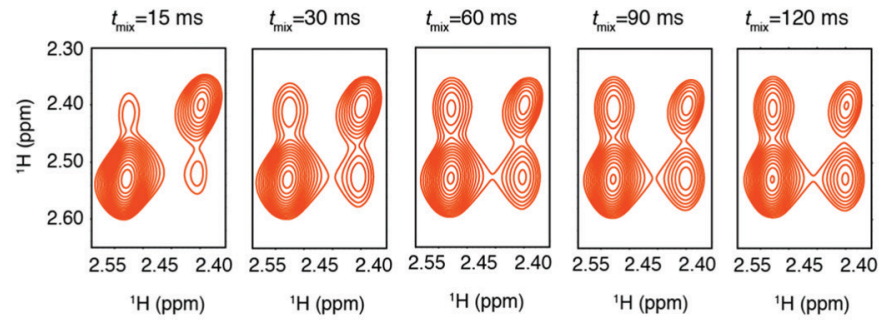

B

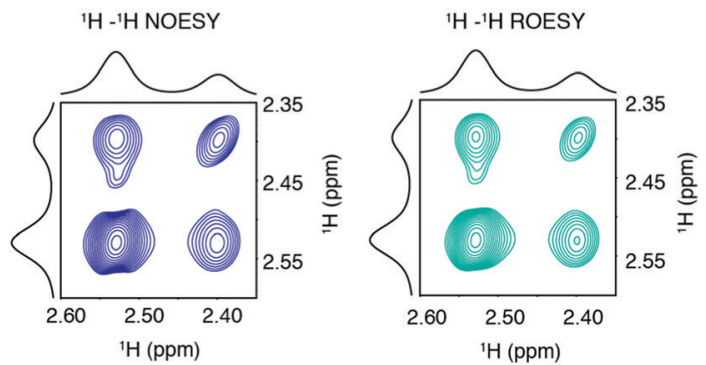

D

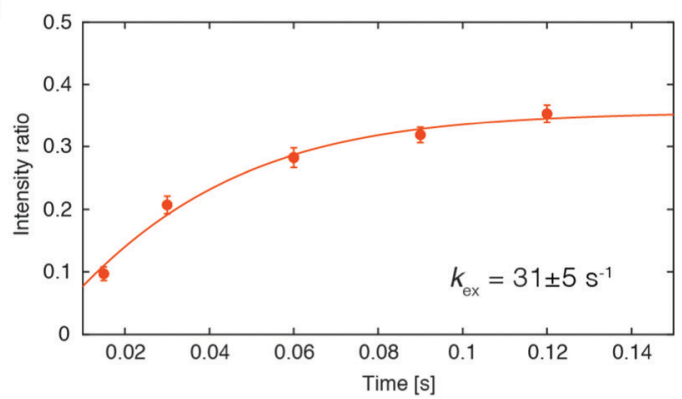

Fig. 3 Detection of chemical exchange of scaffold triethylamine (TEA) molecules between dispersed and condensed phases, through NOESY-type NMR experiments. (A) $2 \mathrm{D}{ }^{13} \mathrm{C},{ }^{1} \mathrm{H}$ plane of an HSQC-NOESY experiment, revealing ${ }^{1} \mathrm{H},{ }^{1} \mathrm{H}$ correlation peaks for the two methylene and methyl ${ }^{1} \mathrm{H}$ signals of TEA molecules from the two phases. The signals belonging to the crowding agent dextran (marked by stars) did not show any correlation peak, consistent with the exclusion of dextran from the condensed phase. (B) $2 \mathrm{D}{ }^{1} \mathrm{H},{ }^{1} \mathrm{H}$ NOESY and ROESY spectra, zoomed over the two methylene signals of TEA originating from the two phases. The same sign of correlation peaks in NOESY and ROESY spectra confirm "chemical exchange" as their source. (C) The methylene region of the ${ }^{1} \mathrm{H},{ }^{1} \mathrm{H}$ EXSY spectra of TEA, measured during the initial stage of phase separation at five different mixing times. (D) Quantitative analysis of the intensity build-up curves provides the exchange rate, $k_{\text {ex }}$, of TEA molecules between the two phases. Error bars are calculated by error propagation based on the spectral noise for each mixing time.

potential client molecule. The choice of HFiP was motivated by its fluorine content, as ${ }^{19} \mathrm{~F}$ nuclei are highly sensitive to the chemical environment and consequently could act as sensitive probes of exchange processes. ${ }^{28}$ After establishing that HFiP $(1.6 \% \mathrm{~m} / \mathrm{v})$ did not perturb the phase separation of TEA (Fig. S1, ESI $\dagger$ ), we monitored how ${ }^{1} \mathrm{H}$ and ${ }^{19} \mathrm{~F}$ NMR signals of HFiP (Fig. 4A) were affected by TEA phase separation. Upon temperature increase and the consequent TEA phase separation, both the methine ${ }^{1} \mathrm{H}$ signal and the trifluoromethyl ${ }^{19} \mathrm{~F}$ signal of HFiP displayed a small downfield shift together with significant signal broadening (Fig. 4B). In addition, a new signal appeared approximately $277 \mathrm{~Hz}$ away from the original peak in the ${ }^{19} \mathrm{~F}$ NMR spectrum. The newly emerged peak originates from HFiP molecules inside the condensed phase, and its exchange between the two phases is expected to be slow on the NMR chemical shift timescale, i.e. slower than $c a .1700 \mathrm{~s}^{-1}$. The presence of HFiP's exchange between the two TEA phases was subsequently supported by $2 \mathrm{D}{ }^{19} \mathrm{~F},{ }^{19} \mathrm{~F}$ NOESY spectra, in which the two ${ }^{19} \mathrm{~F}$ signals showed cross-peaks of the same sign, despite the rapid rotational dynamics of trifluoromethyl groups.

To quantify the kinetics of HFiP exchange between the two phases, we employed the heating-cooling cycles described above and performed $2 \mathrm{D}{ }^{19} \mathrm{~F},{ }^{19} \mathrm{~F}$ EXSY experiments at five different mixing times from 15 to $120 \mathrm{~ms}$ (Fig. 4C and D). Analysis of the intensity build-up curves for the two exchangerelated cross-peaks provided a $k_{\text {ex }}$ of $16 \pm 6 \mathrm{~s}^{-1}$. The obtained $k_{\text {ex }}$ value for the client HFiP is thus nearly half the value observed for the scaffold TEA molecules, suggesting that the exchange processes of the scaffold TEA and the client HFiP molecules are governed by different rate-determining steps.

In cells, the dynamic component exchange between membrane-less organelles and their environment enables their rapid formation and dissolution in response to regulatory signals. ${ }^{19}$ In addition, the rapid recruitment and dynamic exchange of client molecules with the cellular environment are of utmost functional importance for some phase separated cellular bodies. ${ }^{20,21}$ The exchange rates for the scaffold and client molecules are expected to vary over the course of nonequilibrium phase separation processes during which the size of phase-separated droplets, their number and consequently the total interface area between two phase changes (Fig. 4E). ${ }^{4}$ Further changes in the exchange rates may be caused by droplet maturation processes, which affect the internal structure and properties of phase separated droplets. ${ }^{29}$ Previous studies suggest that the phase separation of TEA obeys a nucleationdependent kinetic regime, ${ }^{30}$ resulting in alterations in the exchange rates depending on droplet size and numbers (Fig. 4E). Here, the reversibility of temperature-dependent TEA phase separation allowed us to develop a heating-cooling cycle experimental scheme and specifically target the "initial exchange rate" for both the scaffold TEA and the client HFiP molecules. Based on our results, we argue that it is important to carefully design exchange-related experiments and measure the 


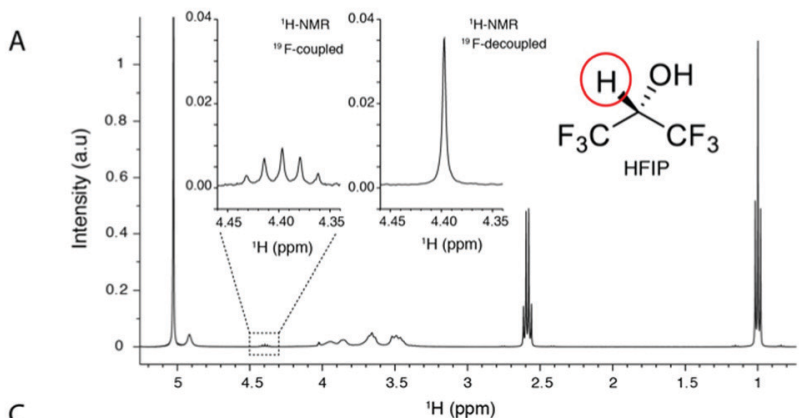

C

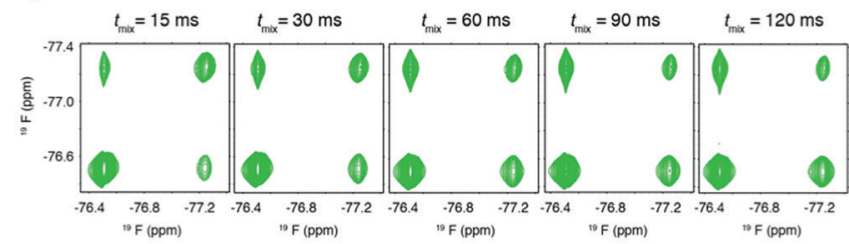

$\mathrm{E}$

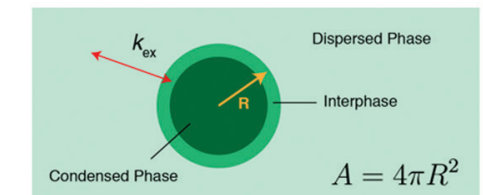

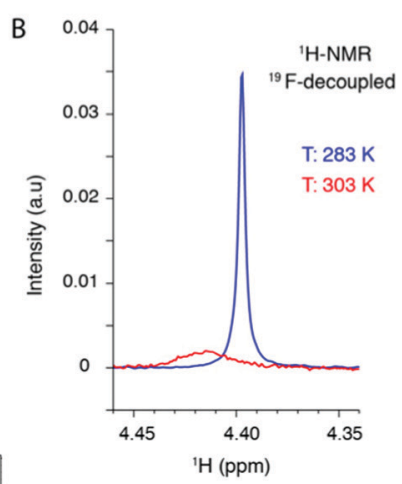

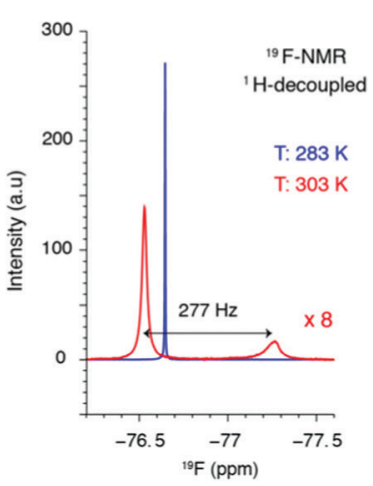

D

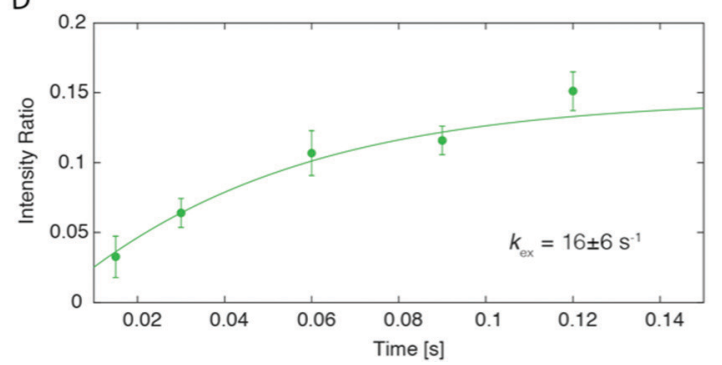

Fig. $4{ }^{19} \mathrm{~F}$-NMR based detection of chemical exchange of a fluorine-containing client molecule, HFiP, between dispersed and condensed triethylamine (TEA) phases. (A) $1 D^{1} \mathrm{H}$ NMR spectrum of the TEA-water sample containing HFiP $(1 \%, v / v)$, before induction of phase separation. The methine proton signal of HFiP is shown in ${ }^{19} \mathrm{~F}$-coupled or decoupled spectra. (B) The effect of TEA phase separation on the methine ${ }^{1} \mathrm{H}$ (left panel) and trifluoromethyl ${ }^{19} \mathrm{~F}$ (right panel) signals of HFiP. (C) ${ }^{19} \mathrm{~F},{ }^{19} \mathrm{~F}$ EXSY spectra of HFiP, measured during the initial stage of TEA phase separation at five different mixing times, displaying mixing time-dependent intensity build-up of the correlation peak. (D) Quantitative analysis of intensity build-up curves yields the exchange rate, $k_{\text {ex }}$ of client molecules between two phases. Error bars are calculated by error propagation based on the spectral noise for each mixing time. (E) Schematic representation of the $k_{\mathrm{ex}}$ dependence on the size of droplets (e.g. their radius, $R$ ), hence the surface area, $A$, of the interphase between the two phases.

exchange rates at a clearly defined kinetic stage of the phase separation process. This will enable monitoring how various regulatory factors, e.g. (bio)chemical regulatory signals, alter the component exchange processes. Thus, comparison of the exchange rates between different client molecules and droplets provides mechanistic insights into the selective recruitment and spatial distribution of biomolecules inside cells.

Our results provide an example of how a multi-spin NMR approach can be employed in detecting and quantifying exchange processes during phase separation. In particular, we showed how a shift from more widely used nuclei such as ${ }^{1} \mathrm{H}$ and ${ }^{13} \mathrm{C}$ to ${ }^{19} \mathrm{~F}$ may be beneficial in such studies. As shown for the client molecule HFiP (Fig. 4), the large sensitivity of ${ }^{19} \mathrm{~F}$ nuclei to the chemical environment leads to a larger value for the chemical shift difference between the signals originated from the two phases $\left(\Delta \omega\right.$, in $\left.\mathrm{s}^{-1}\right)$, and at a given $k_{\mathrm{ex}}$, by shifting the exchange regime further towards the slow-exchange regime (by decreasing the $k_{\mathrm{ex}} / \Delta \omega$ ratio), facilitates exchange rate determination by NMR methods. In addition, the bio-orthogonality of fluorine atoms facilitates their use for biomolecular and biological samples, especially in the context of "exchangebased" small molecule screening against biomolecular targets inside membrane-less organelles. ${ }^{28,31,32}$ The efficacy of drug candidates against biomolecular targets crucially relies on their pharmacokinetic properties ensuring their bio-availability. ${ }^{33}$
Similar to the case of biomolecules located inside membranebound organelles, drugs have to be able to efficiently enter the phase separated membrane-less compartment. Quantitative determination of exchange rates of small molecules between separated phases is thus important. For example, it has been shown that the partitioning of anti-cancer drugs into nuclear condensates is determined by the physicochemical properties independent of their specific targets and influences their activity. ${ }^{34}$ The NMR-based approach developed in the present study enables detection and quantification of the exchange processes between different phases and thereby is an important step towards "exchange-based" screening and optimization of small molecule drug candidates.

\section{Conclusions}

In summary, an NMR-based approach to study component exchange in phase separating systems is presented. Using triethylamine (TEA) as a model system exhibiting reversible temperatureinduced phase separation, we detect and quantify the exchange processes for the scaffold TEA, as well as a client molecule. The developed approach allows the quantitative determination of the effects of regulatory factors on exchange processes and establishes 
the basis for exchange-based small-molecule screening against biomolecular targets located inside the condensed phase.

Acronyms and symbols: TEA (triethylamine), HFIP (hexafluoroisopropanol), LLPS (liquid-liquid phase separation), LCST (lower critical solution temperature), LCST (lower critical solution temperature), DIC microscopy (differential interference contrast microscopy), NMR (nuclear magnetic resonance), HSQC (heteronuclear single quantum coherence), NOESY (nuclear Overhauser enhancement spectroscopy), ROESY (rotating frame Overhauser enhancement spectroscopy), EXSY (exchange spectroscopy), $k_{\text {ex }}$ (chemical exchange rate), $\Delta \omega$ (chemical shift difference in $\mathrm{s}^{-1}$ ).

\section{Author contributions}

C. F. P., M. Z. and N. R.-G.: conceptualization, funding acquisition, data curation; C. F. P.: investigation, visualization; C. F. P. and N. R.-G.: methodology, formal analysis; M. Z. and N. R.-G.: supervision; M. Z.: writing - review \& editing; N. R.-G.: writing original draft.

\section{Conflicts of interest}

There are no conflicts to declare.

\section{Acknowledgements}

C. F. P. acknowledges the Deutsche Akademischer Austauschdienst (DAAD) for Research Grants Doctoral programmes in Germany, 2019/20 (Personal ref. No. 91726791); M. Z. was supported by the European Research Council (ERC) under the EU Horizon 2020 research and innovation programme (grant agreement No. 787679). N. R.-G. acknowledges the Deutsche Forschungsgemeinschaft (German Research Foundation, DFG) for research grant RE 3655/2-3.

\section{Notes and references}

1 S. F. Banani, H. O. Lee, A. A. Hyman and M. K. Rosen, Nat. Rev. Mol. Cell Biol., 2017, 18, 285-298.

2 S. Alberti, Curr. Biol., 2017, 27, R1097-R1102.

3 E. Gomes and J. Shorter, J. Biol. Chem., 2019, 294, 7115-7127.

4 H. Falahati and A. Haji-Akbari, Soft Matter, 2019, 15, 1135-1154.

5 S. Elbaum-Garfinkle, Y. Kim, K. Szczepaniak, C. C. Chen, C. R. Eckmann, S. Myong and C. P. Brangwynne, Proc. Natl. Acad. Sci. U. S. A., 2015, 112, 7189-7194.

6 J. Wang, J. M. Choi, A. S. Holehouse, H. O. Lee, X. Zhang, M. Jahnel, S. Maharana, R. Lemaitre, A. Pozniakovsky, D. Drechsel, I. Poser, R. V. Pappu, S. Alberti and A. A. Hyman, Cell, 2018, 174, 688-699.

7 T. Yuwen, A. Bah, J. P. Brady, F. Ferrage, G. Bouvignies and L. E. Kay, J. Phys. Chem. B, 2018, 122, 11206-11217.
8 S. Ambadipudi, J. G. Reddy, J. Biernat, E. Mandelkow and M. Zweckstetter, Chem. Sci., 2019, 10, 6503-6507.

9 L. Emmanouilidis, L. Esteban-Hofer, F. F. Damberger, T. de Vries, C. K. X. Nguyen, L. F. Ibanez, S. Mergenthal, E. Klotzsch, M. Yulikov, G. Jeschke and F. H. Allain, Nat. Chem. Biol., 2021, 17, 608-614.

10 J. C. Fuentes-Monteverde, S. Becker and N. Rezaei-Ghaleh, Protein Sci., 2021, 30, 1315-1325.

11 N. Rezaei-Ghaleh, F. Munari, S. Becker, M. Assfalg and C. Griesinger, Chem. Commun., 2019, 55, 12404-12407.

12 N. L. Fawzi, S. H. Parekh and J. Mittal, Curr. Opin. Struct. Biol., 2021, 70, 78-86.

13 C. P. Brangwynne, C. R. Eckmann, D. S. Courson, A. Rybarska, C. Hoege, J. Gharakhani, F. Julicher and A. A. Hyman, Science, 2009, 324, 1729-1732.

14 P. R. Banerjee, A. N. Milin, M. M. Moosa, P. L. Onuchic and A. A. Deniz, Angew. Chem., Int. Ed., 2017, 56, 11354-11359.

15 L. Mediani, F. Antoniani, V. Galli, J. Vinet, A. D. Carra, I. Bigi, V. Tripathy, T. Tiago, M. Cimino, G. Leo, T. Amen, D. Kaganovich, C. Cereda, O. Pansarasa, J. Mandrioli, P. Tripathi, D. Troost, E. Aronica, J. Buchner, A. Goswami, J. Sterneckert, S. Alberti and S. Carra, EMBO Rep., 2021, 22, e51740.

16 S. F. Banani, A. M. Rice, W. B. Peeples, Y. Lin, S. Jain, R. Parker and M. K. Rosen, Cell, 2016, 166, 651-663.

17 S. Ranganathan and E. Shakhnovich, J. Phys. Chem. B, 2021, 125, 5035-5044.

18 K. M. Ruff, F. Dar and R. V. Pappu, Proc. Natl. Acad. Sci. U. S. A., 2021, 118, e2017184118.

19 Y. Shin and C. P. Brangwynne, Science, 2017, 357, eaaf4382.

20 L. Galganski, M. O. Urbanek and W. J. Krzyzosiak, Nucleic Acids Res., 2017, 45, 10350-10368.

21 I. A. Sawyer, D. Sturgill, M. H. Sung, G. L. Hager and M. Dundr, BioEssays, 2016, 38, 1197-1208.

22 F. Kohler and O. K. Rice, J. Chem. Phys., 1957, 26, 1614-1618.

23 B. J. Hales, G. L. Bertrand and L. G. Hepler, J. Phys. Chem., 1966, 70, 3970.

24 A. N. Campbell and E. M. Kartzmar, Can. J. Chem., 1969, 47, 619.

25 S. Kajimoto, N. H. Seong, H. Fukumura and D. D. Dlott, Photochem. Photobiol. Sci., 2014, 13, 891-897.

26 R. Behrends, T. Telgmann and U. Kaatze, J. Chem. Phys., 2002, 117, 9828-9837.

27 D. V. Fenby, Z. S. Kooner and J. R. Khurma, Fluid Phase Equilib., 1981, 7, 327-338.

28 J. L. Kitevski-LeBlanc and R. S. Prosser, Prog. Nucl. Magn. Reson. Spectrosc., 2012, 62, 1-33.

29 M. Feric, N. Vaidya, T. S. Harmon, D. M. Mitrea, L. Zhu, T. M. Richardson, R. W. Kriwacki, R. V. Pappu and C. P. Brangwynne, Cell, 2016, 165, 1686-1697.

30 J. Hobley, S. Kajimoto, A. Takamizawa, K. Ohta, Q. Tran-Cong and H. Fukumura, J. Phys. Chem. B, 2003, 107, 11411-11418.

31 A. Boeszoermenyi, B. Ogorek, A. Jain, H. Arthanari and G. Wagner, J. Biomol. NMR, 2020, 74, 365-379. 
32 C. R. Buchholz and W. C. K. Pomerantz, RSC Chem. Biol., 2021, 2, 1312-1330.

33 J. P. Hughes, S. Rees, S. B. Kalindjian and K. L. Philpott, Br. J. Pharmacol., 2011, 162, 1239-1249.

34 I. A. Klein, A. Boija, L. K. Afeyan, S. W. Hawken, M. Fan, A. Dall'Agnese, O. Oksuz, J. E. Henninger, K. Shrinivas,
B. R. Sabari, I. Sagi, V. E. Clark, J. M. Platt, M. Kar, P. M. McCall, A. V. Zamudio, J. C. Manteiga, E. L. Coffey, C. H. Li, N. M. Hannett, Y. E. Guo, T. M. Decker, T. I. Lee, T. Zhang, J. K. Weng, D. J. Taatjes, A. Chakraborty, P. A. Sharp, Y. T. Chang, A. A. Hyman, N. S. Gray and R. A. Young, Science, 2020, 368, 1386-1392. 